\title{
Improving Revocation Scheme to Enhance the Performance in Multi-Authority ABE
}

\author{
Shraddha U. Rasal \\ Department of Computer Science \\ Pune University, Maharashtra, \\ India
}

\author{
Bharat Tidke \\ Professor, Department of Computer Science \\ Pune University, Maharashtra \\ India.
}

\begin{abstract}
Traditional system in cryptography allows just sharing of keys between the sender and receiver, for such a technique only the signature storage is provided for the user's public key. But as the number of users increases, it's became a challenging job to have such a certificate storage as well as key distribution, to overcome this Identity Based Encryption (IBE) was proposed, but again it had created the time consuming environment as it was supporting only to one-to-one communication. After IBE Attribute Based encryption (ABE) made possibility to provide multicast communication between users but it was limited to only key policy based encryption as well as could not provide the revocation phenomenon for keys. So this paper aims to develop an existing system using MAMM (Multiple Authority Multiple Mediator) with the use of distributed CP-ABE (Cipher Policy ABE) which enhances the revocation and improves the performance.
\end{abstract}

\section{Keywords}

Cipher-text, Distributed Cipher-text policy, Encryption, Multi-Authority, Multi-Authority Single Mediator.

\section{INTRODUCTION}

Data privacy is the most important feature in today's computer world. In traditional system, data security had done through encryption and decryption using certificates that binds user's keys. Shamir[1] proposed new concept of Identity Based Encryption (IBE) where instead of using the certificates, he had used the user's own id (example: Self ID).To provide an encryption in multicast manner, Fuzzy IBE[3] have been proposed that leads to an Attribute based encryption $(\mathrm{ABE})$, that could encrypts the document for all the users having set of attributes. The level of access control has been maintained for users as well, these all were carried out by single authority. When the numbers of users were huge, multi-authority had a challenging for doing such work. Multi-Authority Attribute Based Encryption had proposed where services provided were almost for distributed system so, Cipher -policy based encryption technique of cryptography was used. The management of all these attributes/keys was done through the concept called "Revocation" which basically proves the expiration for using the level of access that maintains the higher level of data privacy in encryption. Many solutions were proposed and implemented on revocation as well. The existing work extends to make improvement in revocation techniques. IBE scheme was proposed first to eliminate the certificate storage, where as Attribute Base Encryption proposed new security technology that motivates to survey on best encryption techniques for leveled data privacy. Existing work contains problems of using cipher-text Policy Attribute-Based Encryption (CP-ABE) which allows encrypting data under an access policy, specified as a logical combination of attributes. Such cipher-texts can be decrypted by anyone with a set of attributes that fits the policy. This work can be extended using distributed policy along with multiple mediator Further Section 2 consists literature survey followed by research methodology in Section 3 and Section 4 consists implementation details then conclusion.

\section{LITURATURE SURVEY}

\subsection{Identity Based Encryption}

Shamir [1] proposed a new concept called as an Identity Based Encryption (IBE) in 1998 where the practical implementation was done in 2001.He had simplified an issue of certificate storage. The scheme was an ideal for the closed group of users such as multinational companies, large banks since the headquarters of the corporation can serve as key generation centers. An Identity-based encryption (IBE) is an exciting alternative to public-key encryption, as IBE eliminates the need for a Public Key Infrastructure (PKI). The senders using an IBE do not necessarily look up for the public keys and the corresponding certificates of the receivers, the identities (e.g. emails or Self ID) of the latter are sufficient to encrypt. This scheme had created a transparent environment to the users, when Alice sends mail to Bob at bob@lab.com she just encrypts her message using the public key string lbob@lab.com". There is no need for Alice to obtain Bob's public key certificate. When Bob receives the encrypted mail he contacts with Private Key Generator (PKG) which is the third party. Bob authenticates himself to the PKG and obtains his private key from the PKG. Bob can then read his e-mail. Unlike the existing secure e-mail infrastructure, Alice can send encrypted mail to Bob even if Bob has not yet setup his public key certificate. In identity-based e-mail systems the PKG contains Bob's private key [2].

Fig.1 shown below gives the basic view of an Identity Based Encryption. The message is encrypted under the key, transmitted as cipher-text through the exposed channel, and decrypted under the key. The choice of keys is based on truly random variable say $\mathrm{k}$. The encryption key is the user's identity and the decryption key is derived. So here unlike traditional system the separate key channel between users was eliminated, and is replaced by a single interaction with key generation center when the recipient first joins the network [2]. Shamir implemented a way to provide the more secure system through IBE. An identity-based encryption scheme E specified is used. IBE in other way have some limitations which didn't make fully satisfactory work in Encryption of message as it is only limited for one to one communication to provide error tolerance property, new approach is proposed by Sanahi and Waters in 2005 called Fuzzy Identity Based Encryption (FIBE). 


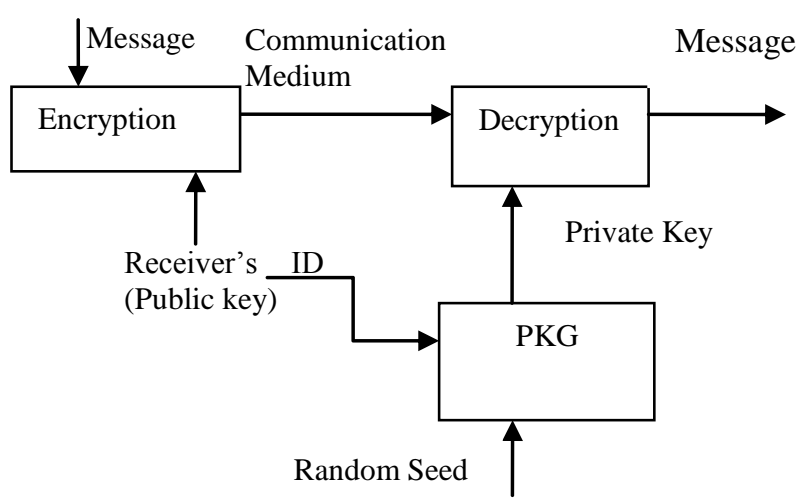

Fig. 1 Identity Based Encryption [1]

\subsection{Fuzzy Identity Based Encryption}

Multicast communication was possible concept proposed (FIBE) by Sahani [3] from IBE which had to two exciting applications. The first was an IBE that used biometric identities and second it could be used for multicast communication. In fuzzy IBE [3][4] identity of a user is viewed as a set of attributes. The name "attributes" given such that number of attributes for each user are located so as to maintain the access level. It had given an idea that how an IBE system encrypts to multiple hierarchical-identities in a collusion-resistant manner implies a forward secure Hierarchical IBE scheme, e.g. \{company, division, department $\}$. It has shown that how their techniques for resisting collusion attacks were useful in attribute-based encryption.

Fuzzy IBE was based on the set of attributes for which the implementation of group of bilinear group was required. Now as group structure was required for the pairing of elements which is a bilinear map [10], the better option for this was proposed by V. Miller [5] in 1985 that is the use of elliptic curves which had an arithmetic structure as well as it is better to fit for solving the problems of traditional system such as Diffie-Hellman or EIGamal. The possible construction of pairing as bilinear map was carried out from two groups say G1 and G2. When groups and pairings are clearly decided then for showing it $(\mathrm{P}, \mathrm{Q})$ are used. Pairings used was nice idea that solved the difficulties of discrete algorithm and rules out the simpler bilinear map for cryptosystem. The group G1 is selected as points on elliptic curves over function $\mathrm{F} q$. The order of G1 was chosen as prime $l$. When $l=q$, there exist an adaptive pairing that sends $\mathrm{G} 1$ to the additive group $\mathrm{G} 2=(\mathrm{F} q$, + ). For such a pairing a key parameter is used which is called as security parameter. When this $r$ (security parameter) was small, the pairing was computed efficiently [6]. Problem occurred when the value became large [3][7]. Two pairings types are defined on elliptic curves that are Weil pairing and Tate pairing. At some value of $r$ Weil pairing was unable to reach the optimum value. On the other hand Tate pairing was used that provided an optimum solution as well has had less cost. For this reason, Frey, Muller and Ruck[8][9][10] proposed in to use it as a replacement for the Weil pairing. Hence the construction of pairing is done as bilinear map on elliptic curve to select a set of attributes.

The preliminaries regarding to the security parameter used on elliptic curve for proper pairing as bilinear map, the multicast communication is possibly carried out through the set of attributes for users hence as variant to the Fuzzy IBE further leads to more advanced Attribute based IBE. For the set of attributes for user, an authority was required in any ways to make the management between the attributes a single authority attributes or multi authority attributes was used depending on the data. Single authority attributes can be easily managed between the users and providers [14][15] but the single authority had some problems such as inefficiency, non scalability and non applicability which were the open problems proposed by M. Pirretti [16], by Shucheng Yu [17], by V.Goyal[18] respectively. The most challenging and interesting job was to manage the security for multi-authority attributes as there were many applications which requires multiple authorities. A student registered with courses across multiple departments may have attributes associated with each of these departments. Designing a multi-authority ABE scheme was an interesting open problem first proposed by Sahani and Waters in [4] and later solved by Melissa Chase [13] in 2007.

\subsection{Multi-Authority Based Encryption}

The focus had made on multi-authority based ABE where each authority having its own procedure to follow the tasks and then combining the results. Multi-authority ABE allowed the sender to specify for each authority a set of attributes say $\mathrm{k}$ monitored by that authority and a number say $d k$ so that the message could be decrypted only by a user who had at least $d k$ for that attributes from each and every authority. It allowed any number of attribute authorities to be corrupted, and guarantee the security of encryption as long as the required attributes could not be obtained exclusively from those authorities and the trusted authority remains honest. But this work again had some problems regarding the collision.

The Sahani and Water [4] had prevented the collusion within authorities, so different keys obtained from any one authority could not combine. For example suppose cipher-text is given which requires attributes from authority 1 and authority 2 . If Alice has all the appropriate attributes from authority 1 and Bob has all the appropriate attributes from authority 2, they still should not be able to combine their keys and decrypt. They had implemented about a single authority that have seen all the attributes requested by a user and had given a secret key, so it had easily re-randomized the secret sharing appropriately. But in multi authority based concept, secrets were necessarily divided between multiple authorities and this had carried out without any communication between the authorities. Here for these purpose two main techniques were used: The first was, every user have a kind of a global identifier (GID) such that: (1) no user can argue on another user's identifier, and (2) all authorities have rights to verify a user's identifier. Thus, the GID could be SSN such that information from identifier would be present when users' attributes were verified. Example given in [13] shows why it is required to have such a credentials: In the first, Bob requests keys for authority 1 and Alice requests keys for authority 2. In the second Bob requests attribute set $A 1$ from authority 1 and attribute set $A 2$ from authority 2 . The global identifier (GID) [13] allowed authorities to distinguish these two scenarios in order to prevent collision.

Each authority acts as pseudorandom function (PRF) which it was used to randomize the secret keys it has given out. A PRF was proving the guaranty that, on the one hand, the secret keys for each user were derived deterministically, but, at the same time they appeared completely random. When a user requested a secret key, the authority computed the PRF on the user's GID and then used the result as the secret in Sahani and Water's key generation. A user with sufficient attributes could then use his secret keys to reconstruct this secret for each authority. Since the outputs of the PRFs would be unique for 
each user, each user has its own set of secrets. Thus, central authority was needed, who knew all of the other authorities' PRFs. For each user, it computed the extra value which, when combined with the secrets the user has reconstructed and resulted in a user-independent system decryption value which allowed the user to decrypt. This idea was worthwhile that broken up the secret throughout multiple authorities based on the user's GID, such that each authority have its own such that each authority was doing its own work independently as only the GID is provided. The use of PRFs mean that each user's secret keys are independent of any other user's keys and collusion is impossible [13]. This was again done through more complex access structure in order to decrypt the ciphertext. Multi-Authority Attribute Based Encryption was worked out along with the access structure using PRF with independent GID. As the organization includes lots of dynamic users there was also necessity of key updating for each user so as to maintain the access control.

\subsection{Key Revocation}

Boneh and Franklin [3] was first suggested simple "Revocation" concept for IBE in random oracle [21] [Appendix B] told about expiration date for keys for maintaining the access control. He had given example about key expiration in IBE such that Alice encrypts e-mail sent to Bob using the public key: \{bob@lab.com\| current-year\}. Bob could use his private key during the current year only. Bob requires new private key once a year from the PKG. Alice did not need to obtain a new certificate from Bob every time Bob refreshes his private key. This approach was carried out by encrypting e-mail for Bob using \{bob@lab.com \| currentdate\}. This forced Bob to obtain a new private key every day. This could be possible in a corporate PKI where the PKG was maintained by the corporation. This approach gives simple key revocation technique: when Bob leaves the company and his key needs to be revoked, the corporate PKG is instructed to stop issuing private keys for Bob's e-mail address. As a result, Bob could no longer read his email. The interesting property was that Alice did not need to communicate with any third party certificate directory to obtain Bob's daily public key. Hence, identity based encryption was very efficient mechanism for implementing ephemeral public keys. Also this approach enabled Alice to send messages into the future: Bob would only be able to decrypt the e-mail on the date specified by Alice. A simple extension to the discussion above enabled to manage user credentials using the IBE system. Suppose Alice encrypts mail to Bob using the public key: lbob@company.com k current-year k clearance=secret". Then Bob will only be able to read the email if it has specific certified credentials. Appropriately, it is easy to revoke user credentials using the PKG. But PKG [25] was not a proper solution due to causing time consuming process. Vipul Goyal [3] proposed an IBE scheme with efficient revocation by combining binary tree data structures with FIBE.His techniques were again applicable to only KP-ABE rather than $\mathrm{CP}-\mathrm{ABE}$. Another approach towards revocation in $\mathrm{CP}-\mathrm{ABE}$ was proxy re-encryption technique that made use of the proxy servers [17]. The proxy servers could be dishonest or could be compromised and hence the scheme was not very secure. In 2011 , with the aim of providing encryption based access control in social networks the authors in [22] proposed the concept of proxy re-keying for minimizing the trust on proxy servers to enable efficient revocation. These approaches were limited to revoking a predefined number of attributes also providing the limitation of existing approaches for revocation in $A B E$ includes inefficiency [3][16], non scalability [17], unreliability and non-applicability to CP-ABE. Furthermore in
2012 Riddhi Mankad et al [25] were combined the multiauthority scheme with revocation concept where she implemented the combinations of authority and mediator with final combination proposed was MASM [25] (Multi-authority Single Mediator) which eliminated the problem about revocation of previous scheme. It is still unable to problem regarding advanced revocation concepts.

Table 1. Survey on Revocation

\begin{tabular}{|c|c|c|c|}
\hline $\begin{array}{l}\text { Proposed } \\
\text { In }\end{array}$ & Approach & Limitations & $\begin{array}{l}\text { Solution } \\
\text { papers }\end{array}$ \\
\hline $\begin{array}{l}\text { Boneh et } \\
\text { al } \\
: 2001[3]\end{array}$ & $\begin{array}{l}\text { First } \\
\text { revocation } \\
\text { concept for } \\
\text { IBE }\end{array}$ & $\begin{array}{l}\text { Hardware } \\
\text { problem and } \\
\text { tamper } \\
\text { resistant } \\
\text { problem }\end{array}$ & $\begin{array}{ll}\text { Pirretti :2006 } \\
{[16]}\end{array}$ \\
\hline $\begin{array}{l}\text { Pirretti et } \\
\text { al } \\
: 2006[16]\end{array}$ & $\begin{array}{l}\text { Revocation } \\
\text { concept for } \\
\text { ABE }\end{array}$ & $\begin{array}{l}\text { Extra load } \\
\text { on PKG }\end{array}$ & $\begin{array}{l}\text { Vipul Goyal } \\
: 2008 \text { [2] }\end{array}$ \\
\hline $\begin{array}{l}\text { Vipul } \\
\text { Goyal } \\
: 2008 \text { [2] }\end{array}$ & $\begin{array}{l}\text { Revocation } \\
\text { in ABE with } \\
\text { Bilinear map } \\
\text { structure. }\end{array}$ & $\begin{array}{l}\text { Unable to } \\
\text { provide } \\
\text { revocation } \\
\text { scheme for } \\
\text { CP-ABE }\end{array}$ & $\begin{array}{l}\text { Shucheng: } \\
2010 \text { [17] }\end{array}$ \\
\hline $\begin{array}{l}\text { Shucheng } \\
\text { et al: } 2010 \\
{[17]}\end{array}$ & $\begin{array}{l}\text { Revocation } \\
\text { with proxy } \\
\text { servers }\end{array}$ & Not secure & $\begin{array}{l}\text { Riddhi } \\
\text { Mankad } \\
2012 \text { [23] }\end{array}$ \\
\hline $\begin{array}{l}\text { Riddhi } \\
\text { Mankad et } \\
\text { al : } 2012 \\
{[23]}\end{array}$ & $\begin{array}{l}\text { First } \\
\text { Combined } \\
\text { Revocation } \\
\text { technique } \\
\text { with Multi- } \\
\text { authority } \\
\text { ABE. }\end{array}$ & $\begin{array}{l}\text { MAMM } \\
\text { scheme is } \\
\text { still an } \\
\text { open } \\
\text { research } \\
\text { Not } \\
\text { applicable } \\
\text { for dynamic } \\
\text { Attributes. } \\
\text { Random } \\
\text { Oracle } \\
\text { problems } \\
\text { Problems } \\
\text { with } \\
\text { distributed } \\
\text { system } \\
\text { revocation. }\end{array}$ & $\begin{array}{l}\text { Qi Li : } 2013 \\
\text { (This paper } \\
\text { solved } \\
\text { problems } \\
\text { about } \\
\text { attribute } \\
\text { access control } \\
\text { ) } \\
\text { Dynamic } \\
\text { attributes } \\
\text { and more } \\
\text { improved } \\
\text { revocation } \\
\text { scheme } \\
\text { through CP- } \\
\text { ABE. }\end{array}$ \\
\hline
\end{tabular}

\section{RESEARCH METHODOLOGY}

The encryption/decryption techniques for any information or message consist of a structure followed by an algorithm. It had implemented sequentially followed by algorithmic steps given below [23].

\subsection{Multi-Authority ABE}

In [13] ABE scheme is explained. It is given that the universe of attributes can be partitioned into $K$ disjoint sets where it is assumed. Each will be monitored by a different authority. It also has one trusted central authority that does not monitor any attributes. Note: In the following we use $A u$ to denote the attribute set of user $u$ and $A C$ to denote the attribute set of a cipher-text. $A k u$ and $A k C$ are the attributes handled by authority $k$ in the attribute sets of the user and the cipher-text respectively. A Multi-Authority ABE system is composed of 
$K$ attribute authorities and one central authority. Each attribute authority is also assigned a value $d k$. The system uses the following algorithms:

Setup: A randomized algorithm which must be run by some trusted party (e.g. central authority) takes as input the security parameter. Outputs a public key, secret key pair for each of the attribute authorities, and also outputs a system public key and master secret key which will be used by the central authority.

Attribute Key Generation: A randomized algorithm run by an attribute authority. Takes as input the authority's secret key, the authority's value $d k$, a user's GID, and a set of attributes in the authority's domain $A k C$. (We will assume that the user's claim of these attributes has been verified before this algorithm is run). Output secret key for the user.

Central Key Generation: A randomized algorithm runs by the central authority. Takes as input the master secret key and a user's GID and outputs secret key for the user.

Encryption: A randomized algorithm runs by a sender. Takes as input a set of attributes for each authority, a message, and the system public key outputs the cipher-text.

Decryption: A deterministic algorithm runs by a user. Takes as input a cipher-text, which was encrypted under attribute set $A C$ and decryption keys for an attribute set $A u$. Outputs a message $m$ if $|A k C \cap A k u|>d k$ for all authorities $k$. Multiauthority along with revocation scheme was implemented whose last step nothing but MASM (Multiple Authority Single Mediator) was proposed by Riddhi Mankad et al [23].

\subsection{MASM}

When multi-authority $\mathrm{ABE}$ was implemented along with revocation, this scheme was used by [23] as their proposed work in encryption of data where it was consisting of a central authority (TA), multiple authorities and a mediator that was so called as MASM (Multiple Authority Single Mediator) as shown in figure 3.1. Every authority has its own set of attributes that are independent on other authority attributes. The mathematical form of such scheme is given as follows [23]:

Global Setup: GP, GS: The trusted authority (TA) will choose a bilinear group $\mathrm{G}$ of prime order $\mathrm{p}$. Let $\mathrm{g}$ be the generator and $\mathrm{e}$ $(\mathrm{g}, \mathrm{g})->\mathrm{G} 1$ be a bilinear map defined on G1. Let $\mathrm{H}:\{0,1\}^{*_{-}}$ $>\mathrm{G}$ be a hash function that maps global identity, GID to a group element and $\mathrm{H} 1:\{0,1\} *_{-}>\mathrm{G}$ that maps string attribute to a group element. Pick two random exponents $\alpha, \beta \in \mathrm{R} Z \mathrm{p}$. The TA generates the global public parameters $\mathrm{GP}=\left\{\mathrm{G}, \mathrm{g},\left(\mathrm{h}=\mathrm{g}^{\beta}\right.\right.$ ), $\left.\mathrm{e}(\mathrm{g}, \mathrm{g})^{\alpha}\right\}$ and $\mathrm{GS}=\left\{\beta, \mathrm{g}^{\alpha}\right\}$.The hash functions $\mathrm{H}, \mathrm{H} 1$ are known only to the authorities.

Authority Setup (GP,Ui)-> PKj, SKj: Each authority $i$ outputs its own public key, PKj and secret key, SKj. For $\forall$ attribute $j$ $\in U i$, choose random exponent $b j \in R Z_{n}$,

$$
\begin{gathered}
P K j=\{[H 1(j)] b j, g b j\} \\
S K j=\{b j\}
\end{gathered}
$$

UserKeyGen(GID,GP, GS)->KO:The trusted authority uses GID and returns the base component of user's(with global Identifier GID)secret key using global secret component GS as below:

$$
K 0=g^{\{(\alpha+r) / \beta\}}
$$

Where $\mathrm{r}=\mathrm{H}(\mathrm{GID})$ and $r \in \mathrm{Z}_{\mathrm{p}}$.
AttributeKeyGen (GID, GP,j,SKj )-> Kuj,Kmj:User request for attribute component of secret key for attribute $j$ to respective authority $i$. This authority makes confirmation about $\mathrm{j} \in U i$ and computes $\mathrm{r}=\mathrm{H}(\mathrm{GID})$. Then it chooses $a j$ randomly and makes keys as $\mathrm{K} u j$ and $K m j$ as ,

$$
K_{u j}=g^{\frac{r}{b j}} H 1(j)^{\frac{a j}{b j}}, K_{m j}=g^{\frac{a j}{b j}}
$$

$K u j$ given to the user and $\mathrm{Kmj}$ is given to mediator.

Encrypt $(M, T, P K j, G P)$->CT: Using access policy $\mathrm{T}$ sender encrypts the message M. It chooses the polynomial $q_{x}$ for each node in access tree having degree $d_{x}=t_{x}-1$ is the threshold value of node $x$. It sets the value of root node $q_{R}(0)=s$, where $s \in R Z_{p}$ and $\mathrm{R}$ is root node. The remaining terms of polynomial are randomly chosen to make a polynomial $q_{x}$ of length $\mathrm{t}$. For all other child node say $\mathrm{z}$, it sets $q_{\mathrm{z}}(0)=\mathrm{q}_{\mathrm{z}}$ ' where $\mathrm{z}$ ' is parent node of $\mathrm{z}$ and algorithm continues assigning the values of similar fashion in a top down manner .Let $y \in Y$ be the set of leaf node and let $\operatorname{att}(y)$ be a function that returns the specific attribute to leaf node $y$. let $j=\operatorname{att}(y)$.

$$
C_{0}=h^{s}, C_{1}=M \cdot e(g, g)^{\alpha s}
$$

For each leaf node, y $\in_{T}$, compute

$$
C_{y}=g^{b j q y(0)}, C_{y}^{\prime}=H 1(j)^{b j q y(0)}
$$

It returns cipher text $\mathrm{CT}$ as $\mathrm{CT}=\left\{\mathrm{T}, \mathrm{C}_{0}, \mathrm{C}_{1}, \mathrm{C}_{\mathrm{y}}, \mathrm{C}_{\mathrm{y}}\right\}$

$M$-Decrypt $(C T, K m j, y, G I D)->C{ }_{y}$ : The mediator takes the cipher text and performs partial decryption using its secret key component and user's (Receiver) GID, provided that the GID is not revoked. If the GID is revoked, then the associated authority needs to issue a new key to the user by performing ReUserKey step as explained. If GID is not revoked, the mediator outputs partially decrypted cipher text C" ${ }_{y}$ and sends it to the receiver. The mediator will stop issuing to the user if the user is revoked.

$$
C^{\prime \prime}{ }_{y}=e\left(C^{\prime}{ }_{y}, K_{m j}\right)
$$

ReUserKey (new GID,GP)->KO:TA verifies the authenticity of the revoked user and issues a new base component of the secret key to him. Thereafter, the user requests for the attribute component of his secret key $\mathrm{K}_{0}$ from individual authorities using AttributeKeyGen step.

$$
K 0=g^{\frac{\alpha+r n e w}{\beta}}
$$

Where $r_{\text {new }}=H\left(\right.$ newGID) and $r_{\text {new }} \in Z_{\text {p. }}$. Thereafter, a user will request attribute component of his secret key by calling AttributeKeyGen function.

$u$-Decrypt $\left(C T, K_{u j}, y, C\right.$ 'j)->A: The receiver takes cipher text, along with his secret key $K_{\mathrm{uj}}$ component and partially decrypted cipher-text, recovered from the mediator to compute $C$," ${ }_{\text {y. }}$ as

$C^{\prime \prime \prime}=e\left(C_{y}, K_{u j}\right), e\left(C_{y}, K_{u j}\right) / e\left(C^{\prime}{ }_{y}, K_{m j}\right)$

$\operatorname{Decrypt}\left(C T, K_{0} A\right)$ - $>M$ : To retrieve the message $\mathrm{M}$, the receiver needs certificate authority, $\mathrm{K} 0$ and $\mathrm{A}$. The receiver decrypts message by feeding certificate authority, K0 and A into the decryption function. The decrypt function for this receiver would have failed had he been revoked or his secret key had not satisfied the policy. 


$$
\frac{e(C 0, K 0)}{A}=e\left(h^{S}, g^{\frac{\alpha+r}{\beta}}\right) / e(g, g)^{r s}
$$

MASM contains single mediator which further inspires in making more improvement in Revocation scheme as well as gives an idea about extending it as MAMM (Multiple Authority Multiple Mediator).

To retrieve $\mathrm{M}, \mathrm{M}=\frac{C 1}{\frac{e(C 0, K 0)}{A}}=M \cdot e(g, g)^{\alpha s} / e(g, g)^{\alpha s}=\mathrm{M}$

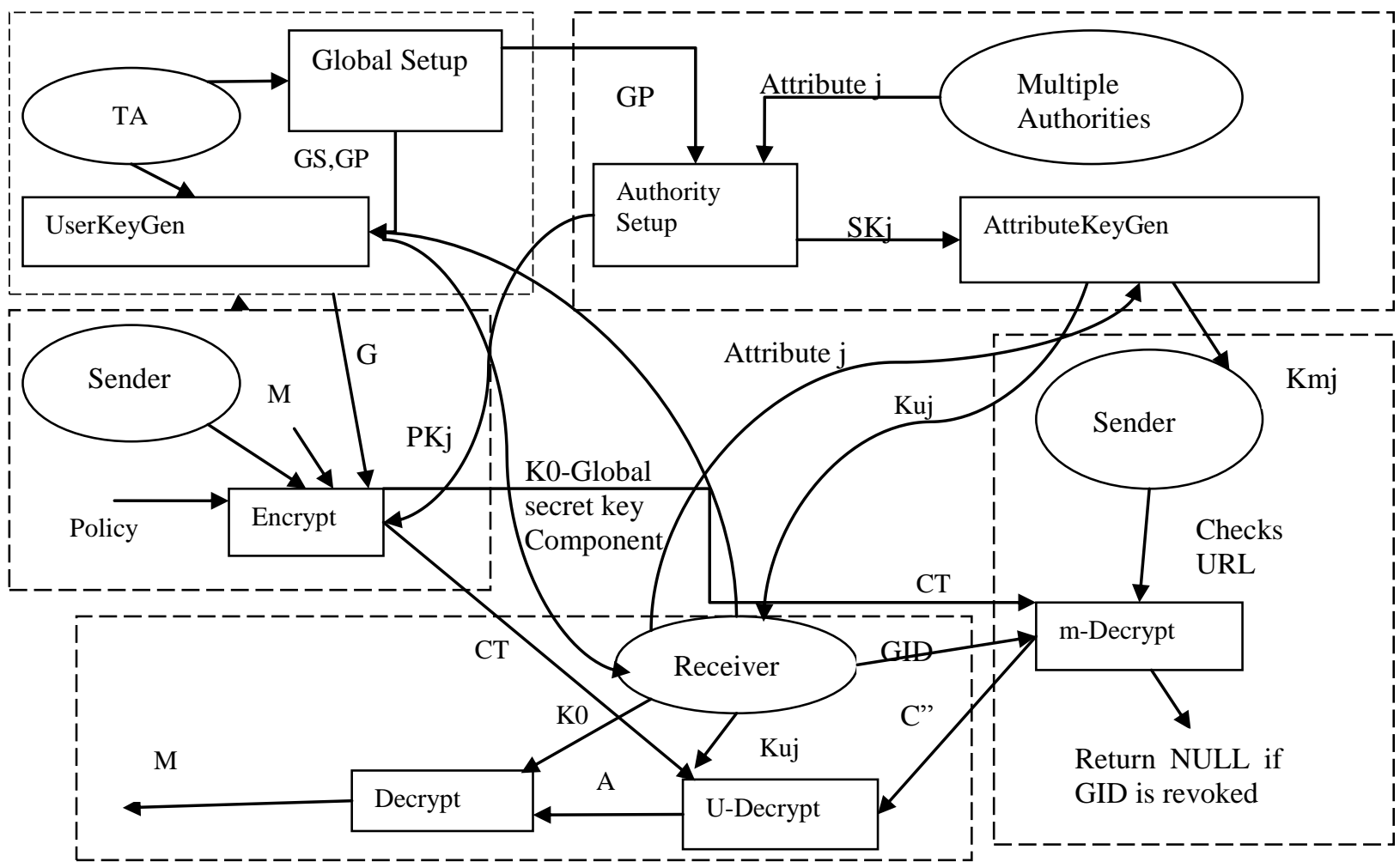

Fig 2: Multiple Authority Single Mediator [23]

\section{IMPLEMENTATION DETAILS}

The logical combination of attributes was used by the traditional CP-ABE (Cipher-text Policy Attribute Based Encryption) scheme. Such cipher-texts can be easily decrypted by anyone as set of attributes that fits the policy. This overall survey up to Multi-authority attribute based Encryption along with Revocation mainly focused on combining the MultiAuthority attribute scheme with revocation. But as it was first model proving combined scheme and hence includes some problems regarding the improvement in revocation techniques due to the use of single mediator. Distributed version of CP$\mathrm{ABE}$ (DABE) scheme allows to extend this single mediator scheme to multiple mediator (Multiple Authority Multiple Mediator) so that the problem of mediator compromising is avoided. The multiple parties are responsible to keep the track of number of arbitrary secrets keys. Distribute CP-ABE contains such multiple attribute authorities to distribute secret keys. So, algorithm for such a scheme is possibly used in multiple mediator which gives basic mathematical steps as is given below.
Terminologies for algorithm [26]:

\begin{tabular}{ll}
\hline Key & Description \\
\hline Pk & Global Key \\
$\mathrm{Mk}$ & Master Key \\
SKa & Secret Key of attribute authority a \\
PKA & Public Key of attribute A \\
SK A,u & Secret Key of Attribute A for User u \\
PKu & Public Key of User u \\
SKu & Secret Key of User u \\
\hline
\end{tabular}

Setup: The initial step gives global setup to produce the $\mathrm{Pk}$ and $\mathrm{Mk}$ as an output.

CreateUser $(P k, M k, u)$ : This function takes input from global setup as $\mathrm{Pk}, \mathrm{Mk}$ and user name $\mathrm{u}$ and outputs the public user key $\mathrm{PKu}$ which is used by the attribute authorities to issue the secret attribute key for $\mathrm{u} \mathrm{SKu}$. This key is used to decrypt the cipher-texts.

CreateAuthority $(P k, a)$ : This is executed by attribute authority with identifier a and creates secret authority key Ska.

RequestAttributePk(Pk,A,SK ): This provides the public attribute key when attribute authorities executed the request by checking the condition as whether A equals a and generates the key PKA otherwise outputs NULL. 
$\left\{P K^{\prime} A:=g^{H s k a(A)}, P K^{\prime \prime} A:=e(g, g)^{y H s k a(A)}\right\}$

RequestAttrbuteSK $\left(P k, A, S k_{a, u} P k_{u}\right)$ : This step check all the conditions of above step and additionally checks whether user $\mathrm{u}$ with public key $\mathrm{PKu}$ is eligible of attribute A. If condition is true then this step outputs SKA , $\mathrm{u}$ for user $\mathrm{u}$ otherwise the algorithm outputs NULL.

$S K_{A, u}:=P K_{u}{ }^{H_{S K} a^{(A)}}=g^{m k u H_{s k a}(A)}$

$\operatorname{Encrypt}\left(P k, M, A, P k_{a 1, \ldots \ldots,} P k_{A N}\right)$ : This step takes an input $\mathrm{Pk}, \mathrm{M}$, access policy $\mathrm{A}, \mathrm{Pk}$ and public keys PKA1,...., PKAn and generates the output as cipher-text CT.

$$
C T_{j}=\left\{E_{j}:=M \cdot\left(\prod_{A \in S_{j}} P K^{\prime \prime}\right)^{R j}\right.
$$

Decrypt: This step takes input as $\mathrm{SKu}, \mathrm{SKA} 1, \mathrm{u}, \ldots . ., \mathrm{SKAn}$ and generates output $\mathrm{M}$.

\section{CONCLUSION AND FUTURE SCOPE}

Literature survey is done based on existing techniques and their implementation which gives an idea that there are still limitations in existing system. This paper shows that algorithm for MASM is implemented which further gives possible construction for MAMM (Multiple Authority Multiple Mediator).Also it can be possibly implemented using distributed version of CP-ABE. So all these approaches gives idea about more scope in encryption techniques under the distributed environment which provides a brief knowledge about future work in the same to enhance the revocation and improves the performance specifically in the area of cloud computing.

\section{REFERENCES}

[1] Adi Shamir,"Identity Based Cryptosystems and Signature schemes" Departments of applied mathematics, 1998.

[2] Alexandra Boldyreva,Vipul Goyal, "Identity- based Encryption with Efficient Revocation",2008.

[3] D. Boneh and M.K Franklin, "Identity-based encryption from the weil pairing". CRYPTO, pages 213-229, 2001.

[4] Sahai and B.Waters, "Fuzzy identity based encryption,"Advances in Cryptology Eu-rocrypt, LNCS, Springer, vol. 3494, pp. 457-473, 2005.

[5] V. Miller, "Use of elliptic curves in cryptography". In H.Williams, editor, Advances in CryptologyCRYPTO'85, volume 218 of Lecture Notes in Computer. Sci., pages 417-428. Springer, 1986.

[6] Antoine Joux, "The Weil and Tate Pairings as BuildingBlocks for Public Key Cryptosystems". Computer Science, Edited by G. Goos, J. Hartmanis, and J. van Leeuwen.

[7] Boneh, B. Lynn, and H. Shacham, "Short signatures from the Weil pairing". In C. Boyd, editor, Proceedings of ASIACRYPT'2001, volume 2248 of Lecture Notes in Computer. Sci., pages 514-532. Springer, 2001.

[8] P. Barreto, H. Kim, B. Lynn, and M. Scott. Efficient algorithms for pairing based cryptosystems. Cryptology 2002. Number 2002/008.

[9] G. Frey, M. Muller, and H.-G. Ruck, "The Tate pairing and the discrete logarithm applied to elliptic curve cryptosystems". IEEE Transactions on Information Theory, 45(5):1717-1718, 1999.
[10] S. D. Galbraith, K. Harrison, and D. Soldera, "Implementing the Tate pairing". Springer-Verlag, 2002.

[11] Piyi Yang, Zhenfu Cao and Xiaolei Dong, "Fuzzy identity based signature," 2008.

[12] Allison Lewko and Brent Waters, "Decentralizing attribute-based encryption," K.G. Paterson (Ed.): Eurocrypt 2011, LNCS, vol. 6632, pp. 568-588, 2011.

[13] Chase, M.: "Multi-authority attribute-based encryption". The Fourth Theory Of cryptography Conference (TCC 2007), LNCS. 4392, 513\{534 (2007).

[14] Waters, B.: "Cipher-text policy attribute based encryption an expressive, Efficient, and provably secure realization". PKC 2011, LNCS, Springer Heidelberg. 6571, (2011).

[15] Luan, I., Milan, P., Svetla, N., Pieter, H., Willem, J.: Mediated cipher-text Policy attribute-based encryption and its application. WISA 2009, LNCS, Springers, Ver- lag. 5932, 309\{323 (2009).

[16] Pirretti, M., Traynor, P, McDaniel, P, Waters, B.: "Secure attribute-based Systems". ACM CCS'06. 6377, 111\{118 (2006).

[17] Shucheng, Yu, Cong Wang, Kui, R., and Wenjing, Lou: "Attribute based data Sharing with attribute revocation". ASIACCS10. (2010).

[18] Alexandra, B., Vipul, G., Virendra, K.: "Identitybasedencryption with Efficient revocation". CCS (2008).

[19] Melissa chase "Multi-authority Attribute Based Encryption", Computer Science Department Brown University Providence, RI 02912.

[20] V. Goyal, O. Pandey, A. Sahai, B. Waters, "Attribute based encryption for fine Grained access control of encrypted data." ACM Conference on Computer and Communications Security, pp. 88-98, 2006.

[21] Bethencourt John, Sahai Amit, Waters Brent, "Ciphertext-policy attribute- Based encryption," IEEE Symposium on Security and Privacy, pp. 321334, 2007.

[22] Sonia Jahid, Prateek Mittal, Nikita Borisov, "Easier: Encryption-based access Control in social networks with efficient revocation," ASIACCS11, March 2011.

[23] Riddhi mankad, Devesh Jinwala "Investigating multi authority attribute-based Encryption with revocation", NIT Surat, 2012.

[24] M. Bellare and P. Rogaway. Random oracles are practical: A paradigm for Designing efficient protocols. In ACM conference on Computer and Communications Security (ACM CCS), pages $62\{73,1993$.

[25] Vipul Goyal," Reducing Trust in the PKG in Identity Based Cryptosystems" Department of Computer Science, University of California, Los Angeles, CRYPTO 2007, LNCS 4622, pp. 430-447, 2007.

[26] Sascha M"uller, Stefan Katzenbeisser, "Distributed Attribute Based Encryption", P.J. Lee and J.H. Cheon (Eds.): ICISC 2008, LNCS 5461, pp.20-36, 2009.Springer-Verlag Berlin Heidelberg 2009. 